\title{
Visual Masking and Visual Integration Across Saccadic Eye Movements
}

\author{
David E. Irwin and Joseph S. Brown \\ Michigan State University \\ Jun-Shi Sun \\ Cornell University
}

\begin{abstract}
SUMMARY
The visual world appears unified, stable, and continuous despite rapid changes in eye position. How this is accomplished has puzzled psychologists for over a century. One possibility is that visual information from successive eye fixations is fused in memory according to environmental or spatiotopic coordinates. Evidence supporting this hypothesis was provided by Davidson, Fox, and Dick (1973). They presented a letter array in one fixation and a mask at one letter position in a subsequent fixation and found that the mask inhibited report of the letter that shared its retinal coordinates but appeared to occupy the same position as the letter that shared its spatial coordinates. This suggests the existence of a retinotopic visual persistence at which transsaccadic masking occurs and a spatiotopic visual persistence at which transsaccadic integration, or fusion, occurs.

Using a similar procedure, we found retinotopic masking and retinotopic integration: The mask interfered with the letter that shared its retinal coordinates, but also appeared to cover that letter. In another experiment, instead of a mask we presented a bar marker over one letter position, and subjects reported the letter that appeared underneath the bar; subjects usually reported the letter with the same retinal coordinates as the bar, again suggesting retinotopic rather than spatiotopic integration across saccades.

In Experiment 3 a bar marker was again presented over one letter position, but in addition a visual landmark was presented after the saccade so that subjects could localize the bar's spatial position; subjects still reported that the letter that shared the bar's retinal coordinates appeared to be under it, but they were also able to accurately specify the bar's spatial position. This ability could have been based on retinal information (the visual landmark) present in the second fixation only, however, rather than spatiotopic visual persistence. Because such a visual landmark was present in the Davidson et al. (1973) experiments, we conclude that their findings can be explained solely in retinotopic terms and provide no convincing evidence for spatiotopic visual persistence.

But the exposure parameters that Davidson et al. (1973) and we used were biased in favor of retinotopic, rather than spatiotopic, coding: The stimuli were presented very briefly just before a saccadic eye movement, and subjects are poor at spatially localizing stimuli under these conditions. Thus, in Experiment 4 we presented the letter array about $200 \mathrm{~ms}$ before the saccade; then, subjects reported that the letter with the same spatial coordinates as the bar appeared under it. This result demonstrates the existence of a spatiotopic memory that combines information from successive fixations, but it does not show whether this memory operates by means of spatiotopic visual fusion.

To determine this, in the last experiment 4 randomly chosen dots from a $3 \times 3$ dot matrix were presented in one fixation, and 4 different dots from the matrix were
\end{abstract}

This research was supported by a grant from the National Science Foundation (BNS 85-19580) to David E. Irwin. Experiments 1-3 were presented at the annual meeting of the Association for Research in Vision and Ophthalmology, Sarasota, Florida, in May 1987.

We thank Brian Butler, John Jonides, and James Zacks for helpful comments on the research; Sam Glucksberg, Geoff Loftus, Sandy Pollatsek, and Adam Reeves for their comments on an earlier version of the manuscript; Mike Tarr and Kyle Cave for assistance with figure preparation; and Elizabeth Dettmer-Radtke for assistance with data collection. David E. Irwin was visiting the Department of Brain and Cognitive Sciences at the Massachusetts Institute of Technology when this article was written, and he gratefully acknowledges their hospitality.

Correspondence concerning this article should be addressed to David E. Irwin, Department of Psychology, Michigan State University, East Lansing, Michigan 48824. 
presented in a second fixation. Subjects reported the location of the missing dot. When the first display was presented just before the saccade (as in Experiments 1-3), subjects accurately specified the missing dot location when the dots were presented to the same region of the retina but not when they were presented in the same place in space. When the first display was presented well before the saccade (as in Experiment 4), subjects performed poorly regardless of retinal or spatial overlap. These results indicate the existence of a short-lived retinotopic visual persistence but provide no support for a spatiotopic visual persistence capable of fusing the contents of successive fixations. We conclude that transsaccadic integration depends instead on an abstract memory that accumulates position and identity information about the contents of successive fixations.

One of the classic questions in perception concerns how people perceive the world as unified, stable, and continuous across changes of eye position. Humans scan the world by means of saccades-fast, ballistic eye movements separated by brief fixations during which the eyes are relatively still. These eye movements create several problems that the human perceptual system must solve to produce a coherent representation of the visual environment. One problem is that visual input is reduced or eliminated during a saccade, so that visual information about the world is registered in isolated glimpses separated in time. A second problem is that the retinal positions of objects in the world change suddenly when the eyes move. But, despite this rapidly changing and temporally discontinuous visual input, people ordinarily perceive the world as a coherent whole. How?

In recent years several investigators have hypothesized that perception of a coherent visual environment is due to the existence of a spatiotopic level of visual persistence that allows the contents of successive fixations to be fused, or integrated, together according to their environmental, rather than retinal, coordinates. For example, McConkie and Rayner (1976) suggested that the new fixation that follows a saccade might override or mask the retinal activity pattern of the prior fixation, while the contents of the two fixations might be spatially fused into a single representation of the stimulus at a higher level in the perceptual system. Similar suggestions have been made by Trehub (1977), Breitmeyer, Kropfl, and Julesz (1982), Jonides, Irwin, and Yantis (1982), Banks (1983), Breitmeyer (1984), and Feldman (1985).

The existence of a spatiotopic level of visual persistence that would allow the fusion of visual information from successive fixations according to environmental coordinates would explain in an intuitively satisfying way the perception of a clear, stable, continuous world across changes in eye position. Unfortunately, attempts to empirically demonstrate the existence of spatiotopic visual persistence have largely been unsuccessful. For example, several investigators have found that subjects are unable to fuse different visual patterns viewed in successive fixations to yield an integrated composite pattern (e.g., Bridgeman \& Mayer, 1983; Irwin, Yantis, \& Jonides, 1983; Jonides, Irwin, \& Yantis, 1983; O'Regan \& Levy-Schoen, 1983; Rayner \& Pollatsek, 1983). Such integration should be possible, it seems, if spatiotopic visual persistence exists. In addition, researchers have shown that changing the visual characteristics of words and pictures (such as letter case or object size) during an eye movement has no disruptive effect on reading, word naming, or picture naming (e.g.,
McConkie \& Zola, 1979; McConkie, Zola, Blanchard, \& Wolverton, 1982; Pollatsek, Rayner, \& Collins, 1984; Rayner, McConkie, \& Zola, 1980). If there was spatiotopic visual persistence, one might expect such changes to interfere with task performance. Other studies once cited as favoring the existence of spatiotopic visual persistence (e.g., Breitmeyer et al., 1982; Jonides et al., 1982; Ritter, 1976; White, 1976; Wolf, Hauske, \& Lupp, 1978, 1980) have been called into question because of various methodological flaws, such as the failure to eliminate phosphor persistence or to monitor eye position (Irwin et al., 1983; Rayner \& Pollatsek, 1983; Sun \& Irwin, 1987).

Perhaps the best evidence in support of spatiotopic visual persistence was provided by Davidson, Fox, and Dick (1973). In one experiment, these investigators presented a letter array in one fixation and a mask at one of the letter positions in a second fixation, and they found that the mask inhibited report of the letter that shared its retinal coordinates even though subjects reported that the mask appeared to occupy the same position as the letter that shared its spatial coordinates. This suggests the existence of two kinds of visual persistence: one retinotopically coded and sensitive to masking and the other spatiotopically coded and integrative with previous fixations. To examine this second memory more closely, in a second experiment Davidson et al. investigated whether two stimuli presented at the same spatial location, but separated by a saccade, would appear to be simultaneously available to a viewer. On each trial a circle and a square were presented just before a saccade; then a vertical and a horizontal line segment were presented in the same spatial area just after the saccade. Subjects had to report whether the line segments spatially overlapped the circle and the square or were adjacent to them. Davidson et al. found that subjects were highly accurate at this task, and, in addition, subjects reported that most of the stimuli seemed to overlap in time at short (i.e., less than 85 $\mathrm{ms}$ ) interstimulus intervals. The two sets of stimuli, however, never appeared to be simultaneously present.

Although the results of the Davidson et al. (1973) experiments are suggestive, they are not completely convincing. The spatial judgments required in the second experiment might have been so easy that they could have been made without spatiotopic visual integration, and the phenomenal reports of apparent spatial overlap across successive fixations were based on the authors' own introspections. We reexamined the Davidson et al. experiments with an eye toward providing a more objective test for the existence of spatiotopic visual persistence. 


\section{Experiment 1}

In Experiment 1 we attempted to replicate the results of the first experiment of Davidson et al. (1973) by using a very similar experimental procedure. Five letters were presented in one fixation, and a mask (or, occasionally, no mask) was presented at one of the letter locations in a second fixation; subjects then attempted to report all five letters that had been presented. The question of interest was whether the mask would interfere with the letter that shared its retinal or spatial coordinates.

\section{Method}

Subjects. The first two authors and two students who were naive to the hypotheses of the experiment participated.

Apparatus. Stimuli were presented on a Hewlett-Packard 1340A point-plotting scope equipped with P31 phosphor. A Digital Equipment Corporation Micro-11/23+ computer controlled stimulus presentation by means of digital-to-analog converters. The computer also recorded the output from a Gulf+Western Applied Science Laboratories Model 210 scleral reflectance eye monitor by means of analogto-digital converters. The eye monitor was configured to record from the right eye only, and it was calibrated to be sensitive only to horizontal movements of the eye. The eye monitor was mounted on eyeglass frames that were held snugly in place on subjects' heads by a headband. During the experiment, subjects were seated $36 \mathrm{~cm}$ from the display scope and used a bite bar with dental impression compound to keep their heads steady. At this viewing distance the display field subtended $20^{\circ}$ of visual angle horizontally and $15^{\circ}$ vertically. The experimental area was dimly illuminated (approximately $0.5 \mathrm{~cd}$ / $\mathrm{m}^{2}$ ), so a red filter and a blue filter were lowered over the face of the display scope to eliminate phosphor persistence visibility; shutter tests similar to those described by Irwin et al. (1983) and by Sun and Irwin (1987) confirmed that no phosphor persistence was visible $5 \mathrm{~ms}$ after stimulus offset.

Procedure. The sequence of events for a typical trial is depicted in Figure 1. Each trial began with a calibration routine during which a calibration point $(+)$ stepped across the display at five locations separated by $1.5^{\circ}$. Each point was presented for $1.5 \mathrm{~s}$, and subjects were instructed to fixate each carefully. Eye position was sampled at each location for $100 \mathrm{~ms}$ (at a rate of once/millisecond) near the middle of the 1.5-s plotting interval. These recordings served as the basis for calibrating the output of the eye monitor against spatial position.

Following calibration, the first fixation point was presented. On rightward-movement trials (as shown in Figure 1) this point was presented at Location 2 of the display, and on leftward-movement trials it was presented at Location 4 of the display. The subject was instructed to fixate this point carefully. After $1.5 \mathrm{~s}$ the first fixation point was extinguished, and the second fixation point, or saccade target, was presented. On rightward-movement trials this point was presented at Location 4 of the display, and on leftward-movement trials it was presented at Location 2 of the display. The subject was instructed to saccade to this target when it was presented. Sampling of eye position (at a rate of once/millisecond) began with the presentation of the saccade target.

To maximize any possible masking effect, we wanted to keep the interval between letter onset and mask onset short. Thus, it was desirable to present the letter array just before the subject initiated the eye movement to the saccade target. To achieve this, following the onset of the saccade target, the program delayed for a time just less than the subject's estimated saccade latency (typically 150-200 $\mathrm{ms}$ ); a staircase procedure tracked this value for each subject through-

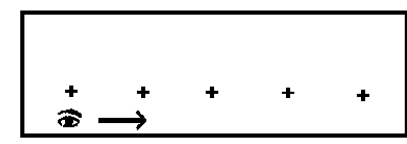

Calibration: Each point presented for $1.5 \mathrm{sec}$, subject fixates each in turn

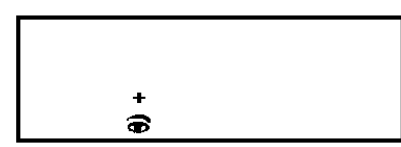

First fixation point presented for $1.5 \mathrm{sec}$

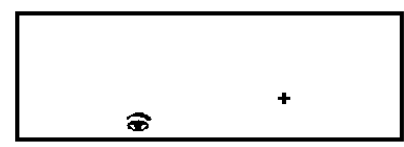

Saccade target presented delay for time just less than subject's estimated saccade latency

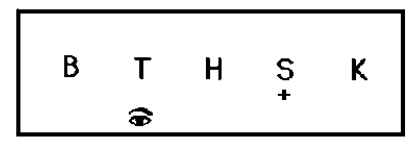

Stimulus display shown for 10 msec just before subject initiates eye movement

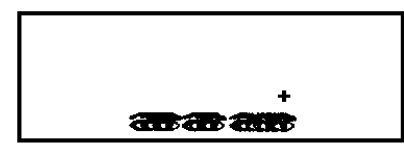

40 msec delay during which eyes move to saccade target

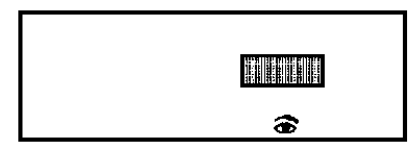

Mask presented for $50 \mathrm{~ms}$ at one letter position, or no mask presented; subject reports letters in display

Figure 1. Schematic illustration of the procedure on a typical trial in Experiment 1 . This is a rightward-movement trial with a mask presented at Location 4.

out the experiment. Following this delay the letter array was presented for $10 \mathrm{~ms}$. The letter array consisted of five uppercase letters drawn from the set of all consonants excluding $y$. Each letter subtended $0.3^{\circ}$ of visual angle horizontally and $0.6^{\circ}$ vertically. One letter was plotted $0.3^{\circ}$ above each of the five calibration locations; the distance between letters was approximately $1.2^{\circ}$.

Following letter presentation, the program checked for the initiation of a saccade; this was defined as a change in eye position velocity greater than $0.05^{\circ} / \mathrm{ms}$ for a 3-ms interval. Once a saccade was detected, the program delayed for $40 \mathrm{~ms}$ before (on most trials) presenting a mask for $50 \mathrm{~ms}$ at one of the locations previously occupied by a letter. The mask consisted of a grid of dots subtending $1.5^{\circ}$ horizontally and $0.6^{\circ}$ vertically. On rightward-movement trials the mask could be presented at Location 3 (thus stimulating what had been Retinal Position 1 in the first fixation), Location 4 (stimulating Retinal Position 2 of the first fixation), or Location 5 (Retinal Position 3 of the first fixation); on leftward-movement trials the mask could be presented at Location 1 (thus stimulating Retinal Position 3 of the first fixation), Location 2 (Retinal Position 4 of the first fixation), or Location 3 (Retinal Position 5 of the first fixation). These locations were used for mask presentation to ensure that the mask would always stimulate both a retinal and a spatial position occupied by a letter. If a mask had been presented at Location 1 following a rightward eye movement, for example, it would have impinged retinally on a point two array locations to the left of any of the letters presented in the fixation preceding the saccade, and it would have had little chance of producing retinal masking. Following mask presentation, the subject attempted to report the five letters that had been presented; these reports were typed into the computer terminal keyboard without regard to the order of the letters in the display. On 
some trials, no mask was presented, but subjects still reported the five letters that had been presented in the display.

Because the purpose of this experiment was to study masking across eye movements, there were certain criteria that a subject's eye movement had to meet for the trial to be acceptable. First, the saccade had to begin after the letter display was extinguished, to ensure that the letters and the mask appeared in separate fixations. To maximize masking, however, we wanted to keep the interval between letter offset and mask onset short; thus, the saccade could begin no later than $30 \mathrm{~ms}$ after letter offset for the trial to be acceptable. The net result of these two restrictions was that, on acceptable trials, the interval between letter offset and mask onset was always between 40 and $70 \mathrm{~ms}$, similar to the intervals used by Davidson et al. (1973). A second set of criteria concerned eye movement accuracy. On each trial the subject was supposed to make a $3^{\circ}$ saccade, so that the mask presented after the saccade would fall on the same retinal position as one of the letters presented before the saccade; if the subject undershot or overshot the saccade target by a wide margin, however, it would be possible for the mask to fall between the retinal positions of the letters. To eliminate this possibility, the subject's saccade had to subtend a range of $2.5^{\circ}$ to $3.5^{\circ}$ for the trial to be acceptable. Because the mask was $1.5^{\circ}$ wide, the mask always covered the retinal position of the letter two locations removed from the mask in the direction opposite the eye movement.

Subjects received feedback after each trial about their eye movement; unacceptable trials were repeated later in the block of trials. Each subject completed 12 blocks of 8 acceptable trials each; these 8 trials consisted of 4 rightward-movement trials and 4 leftward-movement trials. Of these 4 trials, 3 contained masks (at Locations 3, 4, and 5 on rightward-movement trials and at Locations 1, 2, and 3 on leftward-movement trials), and 1 contained no mask. Subjects usually required $30-40$ trials to produce 8 acceptable trials (i.e., approximately $75 \%$ of all trials had to be repeated), primarily because of the saccade's occurring too early or too late relative to letter presentation. Data collection was spread over a period of several days, with each subject usually completing 2-3 blocks of trials per day.

\section{Results and Discussion}

Subjects made five responses on each trial, corresponding to the five letters that they thought had been presented in the letter array. Response omissions were not allowed. The accuracy of these responses was scored without regard for position; in other words, order of letter report did not need to correspond to order of the letters in the array. Hence, only response intrusions (i.e., reports of letters not contained in the array) were counted as errors. The overall error rate, averaged across subjects and conditions, was $40 \%$.

To determine the locus of any possible masking effects, the following comparisons were made. For rightward-movement trials the percentage of correct reports for each array location was calculated for four conditions: no-mask presentation, mask presentation at Location 3, mask presentation at Location 4, and mask presentation at Location 5. Similarly, for leftward-movement trials the percentage of correct reports for each array location was calculated when no mask was presented and when the mask was presented at Locations 1, 2, and 3. Masking effects were then determined by subtracting the percentage of correct reports at each array location when a mask was presented somewhere from the percentage of correct reports at each location when no mask was presented. This yielded three masking effect functions (one for each mask location) for each eye movement direction. Figure 2

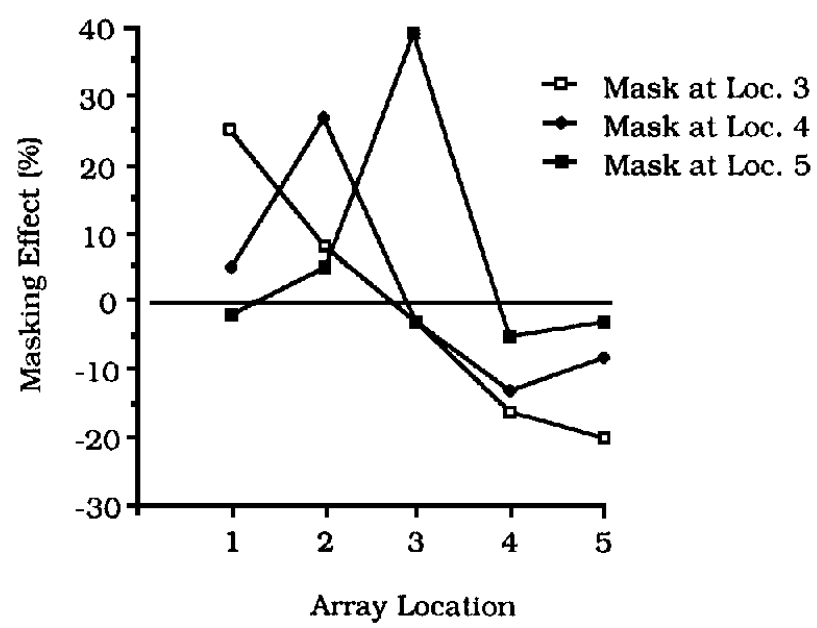

Figure 2. Experiment 1: Masking effect at each array location as a function of mask location on rightward-movement trials. Masking effects were determined by subtracting percentage correct report at each array location when a mask was presented somewhere from percentage correct report at each location when no mask was presented. (Loc. $=$ Location.)

shows these functions for the rightward-movement trials, and Figure 3 shows the functions for the leftward-movement trials, averaged over subjects. The functions for individual subjects were very similar to these averaged functions.

Figures 2 and 3 show that a large masking effect was found only for the array location two positions removed from the mask in the direction opposite the eye movement; this corresponds to the array location having the same retinal coordinates as the mask. The statistical significance of these effects was determined by entering the masking effect results for the individual subjects into a $3 \times 5$ (Mask Location $\times$ Array Location) analysis of variance (ANOVA) for each eye move-

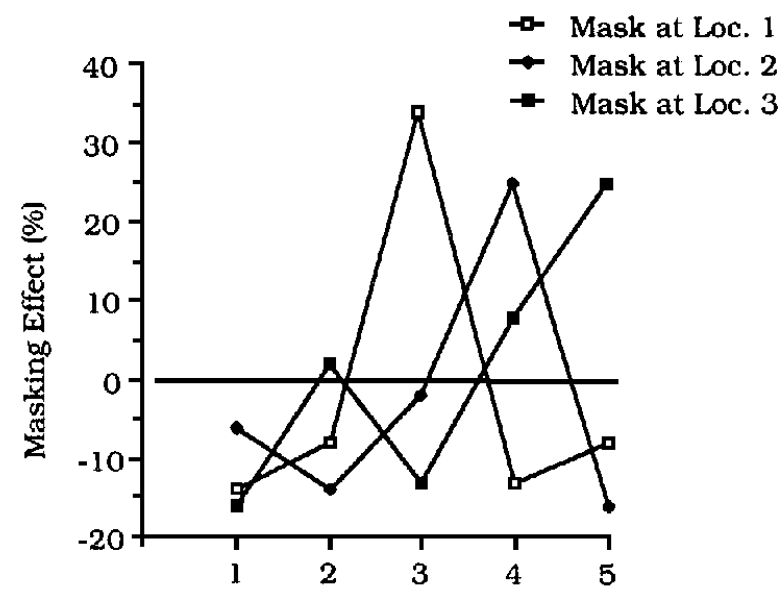

Array Location

Figure 3. Experiment 1: Masking effect at each array location as a function of mask location on leftward-movement trials. (Loc. = Location.) 
ment direction. These analyses revealed significant interactions between mask location and array location for rightward movements, $F(8,24)=8.1, p<.001, M S_{\mathrm{e}}=.015$, and for leftward movements, $F(8,24)=10.9, p<.001, M S_{\mathrm{e}}=.013$. Planned comparisons on these interactions showed that for rightward-movement trials there was a significant masking effect at Location 1 when the mask was presented at Location 3 , at Location 2 when the mask was presented at Location 4, and at Location 3 when the mask was presented at Location 5. For leftward-movement trials there was a significant masking effect at Location 3 when the mask was presented at Location 1, at Location 4 when the mask was presented at Location 2, and at Location 5 when the mask was presented at Location 3. No masking effect occurred for the array location having the same spatial coordinates as the mask, regardless of direction of eye movement. Somewhat surprisingly, there was actually an accuracy facilitation for some array locations on trials in which a mask was presented. For example, accuracy at reporting the letter in Location 5 on rightward-movement trials was $20 \%$ higher when a mask was presented at Location 3 than when no mask was presented anywhere. This accuracy facilitation is most likely due to encoding and output limitations in the no-mask condition. For example, on rightward-movement trials subjects were fixating Location 2 when the letter array was presented, so they were most likely to encode and report the letters at and near Location 2 and less likely to encode and report the letters at Locations 4 and 5 . When a mask was presented, however, one of the letters in Locations 1, 2, or 3 was eliminated by the mask, increasing the likelihood that the letters in Locations 4 and 5 were encoded and reported.

To summarize, like Davidson et al. (1973), we found that masking across saccades is retinotopic. Although the primary purpose of this experiment was to study only masking-and not integration-across eye movements, our subjects reported informally that the mask appeared to cover the letter whose report was interfered with; this disagrees with the finding of Davidson et al. that the mask appeared to be in one position but had its effect at another. This issue was addressed further in the next experiment, which was specifically designed to study integration across saccades.

\section{Experiment 2}

The purpose of this experiment was to provide a more objective test for the influence of spatiotopic visual persistence in integration across eye movements. The experimental procedure was very similar to that used in Experiment 1, except that instead of presenting a mask at one of the letter positions after the saccade, we presented a bar marker over one of the positions. The subjects' task was to report the letter over which the bar was presented. The question of interest was whether subjects would report the letter that was spatially coincident with the bar, retinally coincident with the bar, or some combination of the two. If spatiotopic visual persistence exists and if it mediates the integration of visual information across saccades, we expect subjects would respond on the basis of spatial coincidence; that is, they would report the letter that occupied the spatial position that the bar marker occupied.

\section{Method}

Subjects. The 4 subjects in Experiment 1 participated in this experiment.

Apparatus. The apparatus used in Experiment 1 was used in this experiment.

Procedure. The experimental procedure was identical to that of Experiment 1 with three exceptions. First, to eliminate response intrusions, the stimulus display always consisted of the letters $C, F$, $M, S$, and $X$ in random order. Second, instead of presenting a mask after the saccade, we plotted a bar marker $\left(0.6^{\circ}\right.$ wide $) 0.3^{\circ}$ above one of the letter positions for $50 \mathrm{~ms}$. Third, subjects were instructed to report the one letter that they saw underneath the bar, instead of all the letters in the display.

We used the criteria for eye movement timing and accuracy that were used in Experiment 1; each subject completed eight blocks of 12 acceptable trials each. Of these trials 6 involved rightward eye movements, and 6 involved leftward eye movements. On rightwardmovement trials these 6 trials were composed of two presentations of the bar marker over Letter Positions 3, 4, and 5; on leftwardmovement trials the bar was presented twice over Letter Positions 1, 2 , and 3 .

\section{Results and Discussion}

On each trial subjects reported the one letter that they perceived to be under the bar marker. Figure 4 shows the location of the letter, relative to the actual location of the bar marker, that each subject reported as being under the bar. Averaged over subjects, on $58 \%$ of the trials the letter reported as being under the bar had actually been presented two array locations away from the bar in the direction opposite the eye movement; this corresponds to the array location or letter

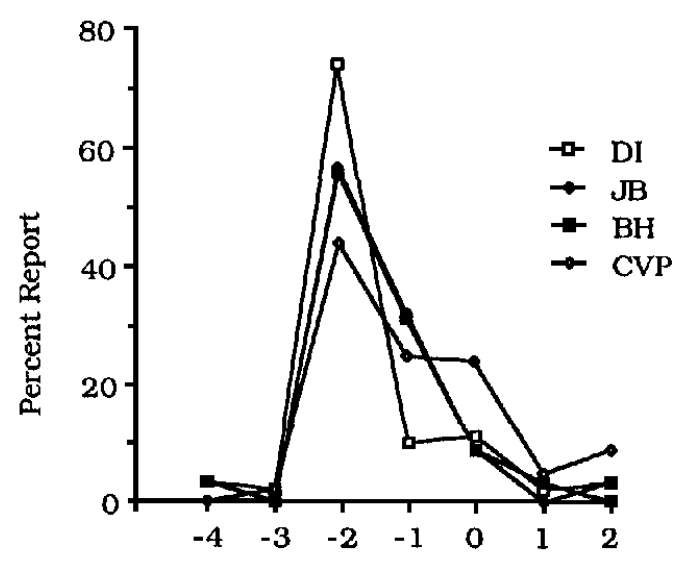

Letter-Bar Distance

Figure 4. Experiment 2: Percentage of trials in which letters at various distances from the bar marker were reported as appearing underneath the marker. The results are shown for each of four subjects. Letter-bar distance refers to the location of letters in the array relative to the actual location of the bar marker; negative distances refer to directions opposite the eye movement, and positive distances to directions toward the eye movement. A letter-bar distance of 0 corresponds to the letter having the same spatial position as the bar marker, and a letter-bar distance of -2 corresponds to the letter having the same retinal position as the bar marker. (DI, JB, $\mathrm{BH}$, and $\mathrm{CVP}=$ the 4 subjects.) 
position having the same retinal coordinates as the bar. On only $13 \%$ of the trials did subjects report the letter that was actually spatially coincident with the bar. A one-way ANOVA showed that there were significantly more retinally coincident reports than reports of any other kind. The $45 \%$ difference between retinally coincident and spatially coincident reports was significant at the .005 level. Although small, the percentage of spatially coincident reports (13\%) was significantly greater than $0(p<.05)$; furthermore, there were a significant number $(24 \%)$ of -1 responses (i.e., reports of the letter between the retinally coincident and spatially coincident letter). Discussion of these latter two results is deferred until Experiment 4.

For now, the most important result of Experiment 2 is that when a letter array was presented in one fixation and a bar marker was presented over one of the letter positions in a second fixation, subjects usually perceived the bar marker to coincide with the letter that shared its retinal, rather than spatial, coordinates. There was little evidence suggesting that visual information presented in successive fixations is integrated according to environmental or spatiotopic coordinates. Thus, this experiment provides little support for the kind of spatiotopic visual persistence reported by Davidson et al. (1973).

There was, however, a potentially important procedural difference between our experiments and the experiments of Davidson et al. (1973). In the Davidson et al. experiments, either the first fixation point or the saccade target was always present; in our experiments, however, the saccade target disappeared after the subject had completed his or her eye movement. It is conceivable that the subjects in the Davidson et al. experiments were able to use the saccade target as a visual landmark to aid their spatial integration and that if we provided subjects with a similar landmark we would obtain evidence for spatiotopic integration. This possibility was investigated in the next experiment.

\section{Experiment 3}

Experiment 3 used the same procedure that Experiment 2 did, with the exception that the saccade target remained present even after the saccade was completed. Because subjects always knew the location of the saccade target (i.e., Location 4 on rightward-movement trials and Location 2 on leftward-movement trials), they could use this information to spatially localize the position of the bar marker. To ensure that they did this accurately, on each trial subjects were asked to report the spatial position of the bar, in addition to reporting which letter appeared to be underneath the bar.

\section{Method}

Subjects. The subjects in Experiments 1 and 2 participated in this experiment.

Apparatus. The apparatus used in the first two experiments was used in this experiment.

Procedure. The sequence of events on each trial was identical to that of Experiment 2 with the exception that the saccade target $(+)$ remained present even after the subject had saccaded to it. Subjects were instructed to report the one letter that they saw underneath the bar and the location (i.e., 1-5) of the bar.
The criteria regarding eye movement timing and accuracy used in the previous experiments were used again. As in Experiment 2, each subject completed eight blocks of 12 acceptable trials each, and these trials were balanced with respect to eye movement direction and bar location.

\section{Results and Discussion}

Subjects were quite accurate at reporting the true spatial position of the bar, reporting the correct location on $78 \%$ of the trials. But Figure 5 shows that their reports of which letter location coincided with the bar were unchanged by the constant presence of the saccade target; on $63 \%$ of the trials subjects reported that the letter that was retinally coincident with the bar appeared to be underneath it, and on only $10 \%$ of the trials was the spatially coincident letter reported as appearing underneath the bar. When we considered only those trials in which the bar marker was accurately localized, these values were $58 \%$ and $11 \%$. As in Experiment 2, a one-way ANOVA showed that there were significantly more retinally coincident reports than reports of any other kind. The 53\% difference between retinally coincident and spatially coincident reports was again significant at the .005 level; furthermore, although there were a significant number of -1 responses (23\%), the percentage of spatially coincident reports (10\%) was not significantly different from 0 . So, as in Experiment 2 , there was little evidence for the existence of spatiotopic visual integration across saccades, even when visual landmarks that might aid spatial coding were present.

Although the 4 subjects who participated in Experiments 2 and 3 uniformly reported on the basis of retinal, rather than spatial, coincidence, 1 naive subject (CVP) did make many more spatial responses than did the other 3 subjects. To examine whether this subject's spatial responses were due to fusion of visual information from successive fixations, we had her complete eight more blocks of trials like those of Experiment 3 but under a new set of instructions. Specifically, the

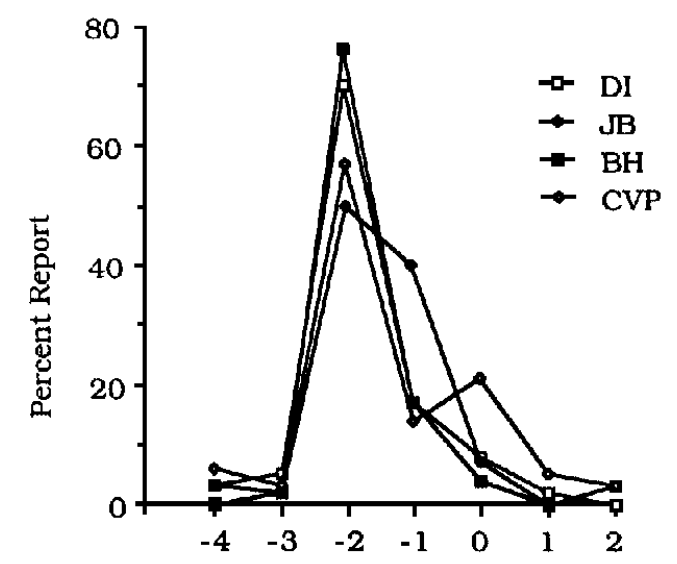

Letter-Bar Distance

Figure 5. Experiment 3: Percentage of trials in which letters at various distances from the bar marker were reported as appearing underneath the marker, for each of 4 subjects. (DI, JB, BH, and CVP $=$ the 4 subjects.) 
subject was instructed to report the letter that appeared to be under the bar, then to report whether the bar and the letters appeared to be simultaneously present (as one would expect them to appear if fusion was occurring). Under these conditions, the subject made $60 \%$ retinally based responses and $18 \%$ spatially based responses, roughly equivalent to her results for Experiment $2(44 \%$ vs. $24 \%)$ and Experiment 3 ( $57 \%$ vs. $21 \%$ ). Most important for present purposes, on $72 \%$ of her retinally based responses the subject judged the bar and the letters to be simultaneously present, but on only $29 \%$ of her spatially based responses did the subject judge the bar and the letters to be simultaneously present. Thus, it seems unlikely that this subject's spatial responses were caused by spatiotopic fusion of visual information from successive fixations.

To summarize, the results of the first 3 experiments provide little support for the existence of a spatiotopic level of visual persistence capable of mediating the integration of visual information across saccadic eye movements. Rather, both masking and integration appear to depend on retinotopic coordinates. But how can the results of the present research be reconciled with those of Davidson et al. (1973)? Experiment 3 suggests a likely explanation. This experiment showed that subjects could accurately report the spatial location of the bar marker even though its integration with the letter array occurred according to retinotopic coordinates. This is just like the finding of Davidson et al. that subjects could accurately report the spatial location of the mask even though the mask interfered with the letter that shared its retinal coordinates. But the ability of subjects to report the spatial location of the bar marker or the mask does not necessarily provide evidence for spatiotopic integration, because it could have been based on visual information present in the second fixation only, namely, the constantly present saccade target. In other words, our results, and the results of Davidson et al., can be explained solely in retinotopic terms; thus, they provide no convincing support for the existence of spatiotopic visual persistence. Van der Heijden, Bridgeman, and Mewhort (1986) recently made a similar critique of Davidson et al., based on logical and theoretical, rather than empirical, grounds.

However, the experimental conditions used in the present research, as well as in the research of Davidson et al. (1973), were biased in favor of retinotopic, rather than spatiotopic, coding; that is, the stimuli were presented for very short exposure durations, under conditions of low illuminance, just before a saccadic eye movement. Previous work by Matin (1972) and others has shown that spatial coding is poor under these conditions. Furthermore, these experimental conditions made it likely that visible persistence from the first fixation would outlast the duration of the saccadic movement to become fused with the retinal contents of the second fixation. Eriksen and Collins $(1967,1968)$ and Di Lollo $(1977,1980)$ have shown, for example, that two visual patterns presented in two separate frames of time to the same part of the retina become fused together as long as the stimulus onset asynchrony (SOA) between them does not exceed $100 \mathrm{~ms}$. Because the SOA between the letters in the first fixation and the mask or bar in the second fixation varied between 50 and $80 \mathrm{~ms}$ in our experiments, retinotopic visible persistence was most likely responsible for the retinotopic integration that we found. With a longer stimulus exposure or with a longer interstimulus interval between the display in the first fixation and the display in the second fixation (both of these manipulations increase SOA), entirely different results might have been found. In support of this possibility, McRae, Butler, and Popiel (1987), using a procedure similar to Davidson et al.'s, found evidence for spatiotopic masking across saccades when a long delay separated the letter array and the mask.

To investigate this issue further, we conducted an experiment similar to Experiment 3 but in which there was a long SOA between the letter display and the bar marker. To keep the experimental procedures as similar as possible, instead of increasing the duration of the letter display to increase SOA, we kept the duration of the display at $10 \mathrm{~ms}$, as in Experiment 3 , and increased instead the interstimulus interval between letter offset and marker onset. This was accomplished by presenting the letter array well before the saccade occurred, rather than, as in Experiment 3, just prior to the saccade. The effect of this change was to increase the SOA between the letters and the bar marker to approximately $250 \mathrm{~ms}$, rather than $50-80 \mathrm{~ms}$ as in Experiment 3. With an SOA of this length, there should be very little or no retinotopic visible persistence from the first fixation, so retinotopic fusion should not occur. But even though these timing parameters should eliminate retinotopic visible persistence, it is perfectly possible that spatiotopic visible persistence follows a different time course, so that spatiotopic fusion of the two displays will occur instead. So the question of interest is, How will subjects respond to the bar marker in this new experiment? Will they still report on the basis of retinotopic overlap, will they instead report on the basis of spatiotopic overlap, or is there no memory, so that their responses will exhibit a random relationship between the locations of the letters in the first fixation and the location of the bar in the second fixation?

\section{Experiment 4}

\section{Method}

Subjects. The first two authors and one of the naive subjects $(\mathrm{BH})$ participated in this experiment.

Apparatus. The apparatus used in the previous experiments was used in this experiment.

Procedure. As in the previous experiments, before every trial the subject completed a calibration routine; then the first fixation point was presented for $1.5 \mathrm{~s}$. Then the saccade target was presented, but, in addition, the stimulus display was also presented for $10 \mathrm{~ms}$. There then was a delay until the subject made a saccade (this delay was for about $200 \mathrm{~ms}$ ) and then another 40-ms delay to allow the subject's eyes to move to the saccade target. Finally, the bar marker was presented for $50 \mathrm{~ms}$ over one of the letter positions, and the subject reported which letter had appeared underneath the bar and the position of the bar. In sum, the major difference between this experiment and Experiment 3 was that the letters appeared $200 \mathrm{~ms}$ before the eye movement, rather than just before the eye movement. We assumed that under these conditions, subjects would have ample time to code the spatial positions of the letters and any retinotopic visible persistence of the letters would have decayed before the bar marker was presented. 
As in Experiment 3, the stimulus display always consisted of the letters $C, F, M, S$, and $X$ presented in random order. However, because the hypothesis under consideration in this experiment was that subjects would be able to respond according to spatial overlap under these display conditions, we wanted to test all possible display locations regardless of saccade direction. Therefore, unlike in Experiment 3, the bar marker could be presented over any one of the five letter locations regardless of eye movement direction. Each subject completed 10 blocks of 10 acceptable trials each, and these trials were balanced with respect to eye movement direction and bar location.

\section{Results}

Figure 6 shows the location of the letter, relative to the actual location of the bar marker, that each subject reported as being under the bar. Averaged over subjects, on $62 \%$ of the trials the letter reported as being under the bar was the letter that had occupied the same spatial location as the bar; on only $3 \%$ of the trials was the letter that shared the same retinal coordinates as the bar reported. A one-way ANOVA showed that there were significantly more spatially coincident responses than responses of any other kind. The $59 \%$ difference between spatially coincident and retinally coincident reports was highly significant $(p<.001)$. The percentage of retinally coincident reports $(3 \%)$ was not significantly different from 0; but, as in Experiments 2 and 3, there were a significant number of -1 reports $(27 \%)$. Increasing the set of display locations that could be probed on each trial from three to five had no deleterious effects on subjects' ability to localize the bar marker; they reported its correct location on $88 \%$ of the trials.

Thus, presenting the letters well before the saccade, rather than immediately before the saccade, resulted in a spatiotopic pattern of reporting; that is, subjects usually reported that the letter that appeared to be underneath the bar was the letter that had the same spatial coordinates as the bar. This result indicates that some kind of spatiotopic memory does exist across saccades. After all, the subjects' shift from a retinotopic pattern of reporting was not to a random pattern but to a

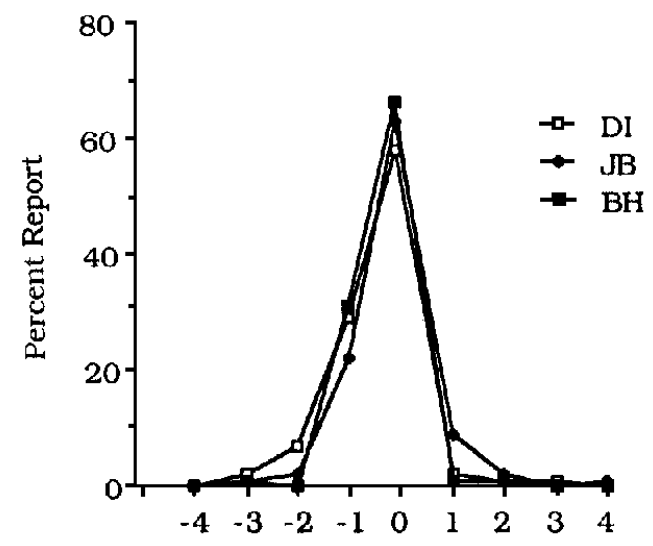

Letter-Bar Distance

Figure 6. Experiment 4: Percentage of trials in which letters at various distances from the bar marker were reported as appearing underneath the marker, for each of 3 subjects. (DI, JB, BH $=$ the 3 subjects.) spatiotopic pattern. The results of Experiments 3 and 4 taken together thus suggest that some transition from retinotopic to spatiotopic coding occurs with the passage of time. Our results are consistent with those of McRae et al. (1987), who found evidence for spatiotopic masking across saccades when a long delay separated the letter array and the mask.

The significant number of -1 and spatially coincident responses found in Experiments 2-3 are most likely due to the transition from retinotopic to spatiotopic coding over time. The number of spatially coincident responses $(10 \%-$ $13 \%$ of all responses) may indicate how much information is completely recoded given a $50-80$-ms SOA between letter onset and marker onset, whereas the -1 responses $(23 \%-24 \%$ of all responses) may reflect information in an intermediate or incomplete state of recoding. The individual differences discussed earlier (i.e., the greater number of spatially coincident responses for CVP than for the other subjects) probably reflect differences in the rate at which different people perform the recoding from a retinotopic to a spatiotopic representation. Additional analyses of CVP's data from Experiments 2 and 3 showed that $81 \%$ of her spatially coincident responses occurred when the bar marker appeared over the first or last location in the array, the most spatially distinct and likeliest starting points for recoding to begin. (The other subjects had too few spatially coincident responses to make this analysis meaningful.) Additional research using a range of SOAs is needed to further elucidate the characteristics of this recoding process.

In sum, the results of Experiment 4 indicate that some spatiotopic memory exists across saccades. But what are the characteristics of this spatiotopic memory? Does it operate by fusing the contents of successive fixations together according to their environmental coordinates to yield an integrated, composite representation of the world, or is it perhaps more abstract in nature, merely containing identity codes and position codes for the elements in each fixation? Phenomenally, performing Experiment 4 seemed quite different from performing Experiments 1-3. In Experiments 1-3, one usually had the impression of seeing the mask or bar superimposed over the letters, as though they were fused together; in Experiment 4 , however, the letters seemed to disappear when the bar was presented, so that even though it was possible to report a letter that was coincident with the bar, the letters and the bar did not appear to be fused together. This introspection suggests that the spatiotopic memory underlying performance in Experiment 4 does not rely on spatiotopic visible persistence and does not operate by fusing visual information from successive fixations. This issue was investigated further in the next experiment.

\section{Experiment 5}

Experiment 5 served two purposes. The first was to verify that the retinotopic integration found in Experiments 1-3 was

\footnotetext{
' Pilot testing showed that spatiotopic integration occurred when only three locations were probed (as in Experiments 2 and 3) as well as when all five locations were probed, so probing five locations instead of three was not the cause of the spatiotopic pattern of results found in this experiment.
} 
caused by retinotopic visible persistence from the first fixation becoming fused with the retinal contents of the second fixation, as we argued in the discussion of Experiment 3. The second was to examine whether spatiotopic visible persistence was responsible for the spatiotopic integration found in Experiment 4. To examine these hypotheses, in Experiment 5 we used a dot-integration task previously used by Di Lollo $(1977,1980)$ to study temporal integration within a single fixation, and by Jonides et al. (1982) and Irwin et al. (1983) to study visual integration across successive fixations. In this task, two halves of a dot matrix, minus one dot, are presented in two frames of time, and the subject must report the location of the missing dot. Performing this task is quite difficult unless the subject is able to fuse the two halves of the dot matrix together into a single composite image. Di Lollo $(1977,1980)$ showed that when the matrix halves were presented to a stationary eye, integration accuracy and the experience of visual fusion decreased as the SOA between the matrix halves increased.

To test for the existence of fusion of visual information across saccades, in Experiment 5 one half of the dot matrix was presented in one fixation, and the second half was presented in a second fixation, after a saccade was made. Sometimes the second half of the dot matrix was presented in the same spatial area as the first half, and sometimes in the same retinal area as the first half, so that the two halves of the matrix overlapped either spatially but not retinally or retinally but not spatially. Sometimes the first half of the dot matrix was presented just before the eyes moved, as in Experiments $1-3$, so that there was a short SOA between the matrix halves, and sometimes the first half of the dot matrix was presented simultaneously with the onset of the saccade target, as in Experiment 4, so that there was a long SOA between the matrix halves.

If the retinotopic masking and integration found in Experiments 1-3 were due to retinotopic fusion of visual information from successive fixations, then accuracy should be better on the retinal overlap trials than on the spatial overlap trials when the Experiment 1-3 timing parameters are used; furthermore, if the spatiotopic integration found in Experiment 4 was due to spatiotopic visual fusion, then accuracy should be better on the spatial overlap trials than on the retinal overlap trials when the Experiment 4 timing parameters are used. On the other hand, if the phenomenal reports described in the discussion of Experiment 4 are correct, there should be little difference between retinal overlap and spatial overlap accuracy when the Experiment 4 timing parameters are used.

\section{Method}

Subjects. The 3 subjects from Experiment 4, plus a new naive subject (CC), participated in this experiment. Another new naive subject also participated but failed to produce accuracies above chance in one of the eye movement conditions and was dropped from the experiment.

Apparatus. The apparatus used in the previous experiments was used in this experiment.

Procedure. Experiment 5 was actually done as two separate experiments, corresponding to the two different sets of timing parame- ters that were used. That is, all subjects completed the set of trials using the Experiment 1-3 timing parameters (hereinafter called short SOA trials) before completing an additional set of trials using the Experiment 4 timing parameters (long SOA trials). The sequence of events on the short SOA trials is shown in Figure 7. Following calibration, the first fixation point was presented for $1.5 \mathrm{~s}$; then the saccade target was presented, and a delay ensued for a time just less than the subject's estimated saccade latency. Then the first half of the dot matrix was presented for $10 \mathrm{~ms}$, centered at the location of the first fixation point. This display consisted of 4 randomly chosen dots from a $3 \times 3$ dot matrix; dots in the matrix were separated from each other by $1.5^{\circ}$ horizontally and $0.75^{\circ}$ vertically. Then, following a 40 $\mathrm{ms}$ delay during which the eyes moved to the saccade target, 4 different dots from the $3 \times 3$ dot matrix were presented for $50 \mathrm{~ms}$, centered either at the location of the first fixation (spatial overlap) or at the location of the saccade target (retinal overlap). So, across successive fixations, 8 of the 9 dots from the $3 \times 3$ matrix were presented, and the subject's task was to report the row and column coordinates of the missing dot. On the spatial overlap trials the two halves of the dot matrix were presented in the same place in space (centered around the first fixation point) but to different regions of the retina (the first half to the fovea and the second half to the periphery); on the retinal overlap trials the two halves of the dot matrix were presented in different spatial areas (Display Locations 2

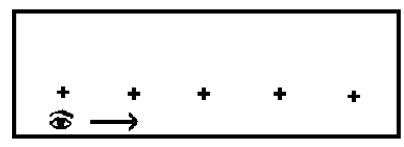

Calibration: Each point presented for $1.5 \mathrm{sec}$, subject fixates each in turn

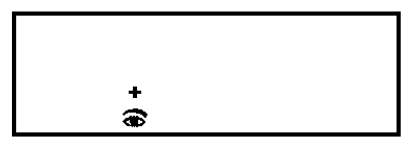

First fixation point presented for $1.5 \mathrm{sec}$

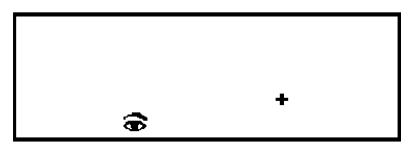

Saccade target presented; delay for time just less than subject's estimated saccade latency

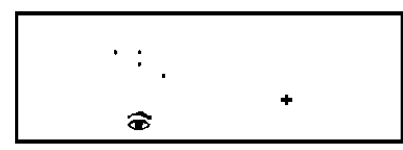

Present first half of matrix for $10 \mathrm{msec}$ just before subject

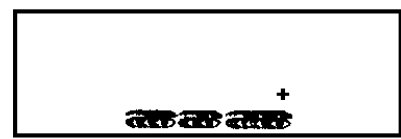

40 msec delay during which eyes move to saccade target

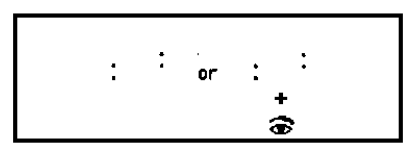

Second half of matrix shown for $50 \mathrm{~ms}$ with spatial or retinal overlap; subject reports location of gap

Figure 7. Schematic illustration of the procedure on short SOA trials in Experiment 5 (rightward-movement trial); the first half of the dot matrix was always presented at the location of the first fixation point, but the second half of the dot matrix was presented either at the location of the first fixation point (spatial overlap) or at the location of the saccade target (retinal overlap). The missing dot location in this example is in the third row, second column. (SOA = stimulus onset asynchrony.) 
and 4 , separated by $3^{\circ}$ of visual angle) but to the same part of the retina (the fovea).

The procedure on the long SOA trials essentially reversed Frames 3 and 4 of Figure 7. On these trials the first half of the dot matrix was presented simultaneously with the onset of the saccade target, there was a delay until the subject initiated a saccade, an additional delay while the eyes moved to the saccade target, and then the second half of the dot matrix was presented, with either spatial or retinal overlap.

The set of criteria regarding eye movement timing and accuracy used in the previous experiments was used again. Each subject completed eight blocks of 12 acceptable trials each, at both the short and long SOAs. These trials were balanced for eye movement direction (left and right) and matrix overlap (spatial and retinal).

Following the completion of these eye movement trials, each subject also completed two no-eye-movement control conditions. In one condition, the two halves of the $3 \times 3$ dot matrix were presented foveally while the subject maintained fixation at one location. In this condition the two halves of the dot matrix overlapped both retinally and spatially, so integration accuracy should have been at its highest; this condition thus served as an upper reference point for integration accuracy in the retinal overlap and spatial overlap eye movement conditions. In the second control condition, one half of the $3 \times 3$ dot matrix was presented foveally, and then the second half was presented $3^{\circ}$ to the side as the subject maintained fixation. In this condition the two halves of the dot matrix overlapped neither retinally nor spatially, so it served as a lower reference point for integration accuracy, or as an indication of how well subjects could figure out the location of the missing dot given no overlap of the matrix halves. Note that the first control mimicked the retinal layout of the retinal overlap eye movement trials (i.e., both halves at the fovea), whereas the second control mimicked the retinal layout of the spatial overlap eye movement trials (i.e., the first half at the fovea and the second half in the periphery). Both control conditions had a short and a long SOA; these SOAs were set equal to the mean of the SOAs that each subject experienced in the eye movement trials. The short and long SOAs, respectively, used for each subject were DI, $70 \mathrm{~ms}$ and $273 \mathrm{~ms}$; JB, $69 \mathrm{~ms}$ and $244 \mathrm{~ms} ; \mathrm{BH}, 70 \mathrm{~ms}$ and $253 \mathrm{~ms}$; and CC, $66 \mathrm{~ms}$ and 215 ms. Each subject completed 50 trials in each control condition at each SOA.

\section{Results}

Table 1 contains the integration accuracy for each subject on retinal overlap and spatial overlap eye movement trials, and on the Retinal + Spatial overlap and no-overlap control trials, under short and long SOA conditions. The results for each SOA condition were analyzed in a separate one-way ANOVA, with condition (four levels) as the sole variable.

Analysis of the short SOA trials revealed a significant effect of condition, $F(3,9)=19.6, p<.001$. Planned comparisons revealed that accuracy on retinal overlap trials was significantly higher than on spatial overlap trials, $F(1,9)=12.2, p$ $<.01$; furthermore, accuracy on the retinal overlap trials was significantly higher than on the no-overlap trials, $F(1,9)=$ $6.3, p<.05$, but accuracy on the spatial overlap trials was not higher than in the no-overlap condition, $F(1,9)=0.9, p>$ .10. So, as predicted, at the short SOA subjects could integrate the two halves of the dot matrix to find the missing dot as long as the two halves overlapped retinotopically; when they overlapped only spatiotopically, subjects' integration accuracy was no better than when the matrix halves did not overlap at
Table 1

Percentage Correct in Each Condition of Experiment 5 at Short and Long SOAs

\begin{tabular}{|c|c|c|c|c|}
\hline Subject & $\begin{array}{l}\text { Retinal } \\
\text { overlap }\end{array}$ & $\begin{array}{l}\text { Spatial } \\
\text { overlap }\end{array}$ & $\begin{array}{c}\text { Retinal + Spatial } \\
\text { overlap }\end{array}$ & $\begin{array}{c}\text { No } \\
\text { overlap }\end{array}$ \\
\hline \multicolumn{5}{|c|}{ Short SOA condition } \\
\hline $\begin{array}{l}\text { DI } \\
\text { JB } \\
\text { BH } \\
\text { CC }\end{array}$ & $\begin{array}{l}58.3 \\
35.4 \\
58.3 \\
25.0\end{array}$ & $\begin{array}{l}22.9 \\
12.5 \\
18.8 \\
10.4\end{array}$ & $\begin{array}{l}56.0 \\
88.0 \\
82.0 \\
64.0\end{array}$ & $\begin{array}{l}26.0 \\
22.0 \\
24.0 \\
24.0\end{array}$ \\
\hline Average & 44.3 & 16.2 & 72.5 & 24.0 \\
\hline \multicolumn{5}{|c|}{ Long SOA condition } \\
\hline $\begin{array}{l}\text { DI } \\
\text { JB } \\
\text { BH } \\
\text { CC }\end{array}$ & $\begin{array}{l}33.3 \\
27.0 \\
29.2 \\
20.1\end{array}$ & $\begin{array}{l}35.4 \\
16.7 \\
27.1 \\
27.1\end{array}$ & $\begin{array}{l}54.0 \\
36.0 \\
40.0 \\
38.0\end{array}$ & $\begin{array}{l}28.0 \\
36.0 \\
52.0 \\
38.0\end{array}$ \\
\hline Average & 27.4 & 26.6 & 42.0 & 38.5 \\
\hline
\end{tabular}

all. These findings support the argument that the results of Experiments 1-3 were caused by retinotopic visible persistence from the first fixation. Accuracy in the retinal overlap condition was significantly lower than in the Retinal + Spatial control condition, however, $F(1,9)=12.3, p<.01$. This was most likely due to disruptive effects of the eye movement on visible persistence (e.g., retinal smear) or perhaps to misalignment of the two halves of the dot matrix in the retinal overlap condition. The Retinal + Spatial control would not suffer from these problems because the eyes remained stationary during stimulus presentation.

Analysis of the long SOA trials also revealed a significant effect of condition, $F(3,9)=4.1, p<.05$. But planned comparisons revealed no significant differences in accuracy between retinal overlap and spatial overlap trials $(F<1)$; between the retinal overlap and no-overlap conditions, $F(1$, $9)=4.2, p>.05$; or between the spatial overlap and nooverlap conditions, $F(1,9)=4.8, p>.05$. If anything, performance in the no-overlap control $(38.5 \%)$ was superior to that in either of the eye movement conditions $(27.4 \%$ and $26.6 \%$ ). Accuracy in the Retinal + Spatial control condition was significantly higher than in either the retinal overlap, $F(1$, $9)=7.2, p<.05$, or the spatial overlap, $F(1,9)=8.0, p<$ .025 , condition, but it was not significantly different from accuracy in the no-overlap control $(F<1)$. In other words, integration accuracy was higher in the no-eye-movement conditions than in the eye movement conditions, but neither retinal nor spatial overlap benefited performance. The superiority of the no-eye-movement control conditions over the eye movement conditions could have been due to practice (i.e., the control conditions occurred after the eye movement conditions), but given that 3 of the 4 subjects had a great deal of experience in previous dot-integration experiments, it seems more likely that the poorer performance in the eye movement conditions was due to the deleterious effects of the 
eye movement on the quality of the visual information available to the subject. One final result worth noting is that accuracy in the retinal overlap and Retinal + Spatial Overlap conditions was lower on the long SOA trials than on the short SOA trials, in accord with Di Lollo's $(1977,1980)$ finding that integration accuracy decreases as SOA increases when the matrix halves are presented to a stationary eye.

In sum, the results of this experiment provide support for the argument that retinotopic visible persistence was responsible for the results of Experiments 1-3, but they provide no support for the existence of spatiotopic visual fusion. It appears unlikely that the spatiotopic reports found in Experiment 4 were due to spatiotopic fusion of visual information across a saccade. This conclusion agrees with subjects' introspections in Experiment 4 that fusion was not apparent. The failure to find spatiotopic fusion with the dot-integration task in this experiment extends previous failures with this task that used different timing parameters (e.g., Bridgeman \& Mayer, 1983; Irwin et al., 1983; Rayner \& Pollatsek, 1983). What distinguishes this experiment from the others is that the timing parameters it used were demonstrably sufficient to produce spatiotopic integration in another setting. So we can now conclude with some confidence that the previous failures to find spatiotopic visual fusion were not due simply to the use of inappropriate timing conditions. It appears instead that the spatiotopic memory uncovered in Experiment 4 is abstract in nature, using schematic visual information or position and identity codes for the elements in a fixation rather than a detailed, photographic representation.

\section{General Discussion}

In this article we reexamined the Davidson et al. (1973) experiments with an eye toward providing a more objective test for the existence of spatiotopic visual persistence. The results of Experiments 1-3 indicated that the Davidson et al. results could be explained solely in retinotopic terms and thus provide no compelling support for the existence of spatiotopic visual persistence. When experimental parameters potentially more conducive to spatiotopic coding were used in Experiment 4, however, evidence for spatiotopic integration was found. The results of Experiment 5 suggest that this spatiotopic integration was not due to the fusion of visual information across saccades, however, but perhaps to some more abstract memory for the contents of successive fixations.

Is an abstract, spatiotopically coded memory sufficient to explain perception of a stable and continuous visual environment across changes in eye position? To perceive the world as stable and continuous, people must somehow overcome two events: retinal smear during the saccade and changes in the retinal positions of objects from one fixation to the next. After all, during fixations themselves, the image on the retina is relatively stable and continuous. Saccadic suppression ordinarily solves the first problem, effectively eliminating perceptual input during the eye movement by means of forward and backward visual masking from the fixations themselves (Campbell \& Wurtz, 1978). An abstract representation of the positions and identities of objects in the environment seems capable of solving the second problem; as long as retinotopic visible persistence from one fixation does not fuse with the retinal contents of a subsequent fixation, knowing where and what objects are in the visual world should be sufficient for one to experience perceptual stability. Experiments 4 and 5 show that the SOAs that ordinarily separate successive fixations do not allow retinotopic visual fusion, and this agrees with everyday perceptual experience: People rarely experience overlapping images when they move their eyes, for example. In sum, it seems to us that one need not postulate the existence of spatiotopic visual persistence to explain perception of a stable and continuous visual world.

Having said this, just to be cautious we should add that even though the results of the present research indicate that spatiotopic visual fusion across saccades does not occur, it is possible that the integration tasks that we used to investigate fusion are simply inappropriate. In our experiments, two different visual patterns (i.e., letters and a bar marker, two halves of a dot matrix) were presented in successive fixations; it is conceivable that the fusion mechanism, if one exists, operates only when there is environmental constancy during a saccade (as there usually is). In fact, Ritter (1976) and Wolf et al. $(1978,1980)$ provided evidence for spatiotopic visual fusion when the subjects' task was to integrate a stimulus or a pattern that remained the same from one fixation to the next. Although these studies have been questioned on methodological grounds (e.g., Irwin et al., 1983; Rayner \& Pollatsek, 1983), the possibilities that they raise deserve and require further investigation. But, in any event, the results of the present research demonstrate that spatiotopic visual fusion does not automatically and arbitrarily occur and that some spatiotopic memory does exist that does not depend on visual fusion.

Furthermore, the conclusion that transsaccadic integration is abstract in nature agrees well with other results in the literature. For example, Rayner et al. (1980) found that a word presented in the visual periphery of one fixation facilitated naming latency for a word viewed in a subsequent fixation if the two words shared the same beginning letters, regardless of letter case; they concluded that abstract letter identities are integrated across saccades during reading. Similarly, Pollatsek et al. (1984) found that visual and conceptual similarity facilitated object identification across changes in eye position, whereas changes in object size had little deleterious effect on performance; they proposed that abstract visual features and object identity codes are combined across fixations.

The notion that transsaccadic integration might be mediated by an abstract memory that codes the positions and identities of the elements of successive fixations to create a coherent representation of the visual environment is also consistent with several theoretical views of how early visual information processing occurs. For example, Hochberg (1968) proposed that the viewer generates a schematic map of the environment and relates successive fixations to it, independently of their retinal coordinates. Kahneman and Treisman (1984) proposed that abstract "object files" might accumulate information about the contents of the environment across changes of eye position. Ullman (1984) proposed that a "marking map" codes where things are in the environment, 
whereas "incremental representations" code information about what they are. All these views seem consistent with the results of the present research.

To conclude, there is spatiotopic persistence across saccades, but it appears not to operate by means of the fusion of visual information across eye movements. Rather, information integration across saccades may be more abstract, depending on schematic visual codes or on the comparison and accretion of identity codes and position codes for objects in the environment. What remains to be determined is just what the characteristics of this spatial memory are. One possibility is that some of the same memories that code position and identity information for the contents of individual fixations are involved in transsaccadic integration as well. For example, Irwin and Yeomans (1986) and Irwin and Brown (1987) found evidence for two kinds of memory for the contents of briefly presented visual displays: One memory maintains a visual representation of the display for a brief time after stimulus offset, and the second memory holds a more durable and perhaps more abstract representation of the display. We are currently investigating what the relationship is between these memories and the spatiotopic memory uncovered in the present research.

\section{References}

Banks, W. P. (1983). On the decay of the icon. Behavioral and Brain Sciences, 6, 14.

Breitmeyer, B. G. (1984). Visual masking: An integrative approach. New York: Oxford University Press.

Breitmeyer, B. G., Kropfl, W., \& Julesz, B. (1982). The existence and role of retinotopic and spatiotopic forms of visual persistence. Acta Psychologica, 52, 175-196.

Bridgeman, B., \& Mayer, M. (1983) Failure to integrate visual information from successive fixations. Bulletin of the Psychonomic Society, 21, 285-286.

Campbell, F. W., \& Wurtz, R. H. (1978). Saccadic omission: Why we do not see a gray-out during a saccadic eye movement. Vision Research, 18, 1297-1303.

Davidson, M. L., Fox, M. J., \& Dick, A. O. (1973). Effect of eye movements on backward masking and perceived location. Perception \& Psychophysics, 14, 110-116.

Di Lollo, V. (1977). Temporal characteristics of iconic memory. Nature, 267, 241-243.

Di Lollo, V. (1980). Temporal integration in visual memory. Journal of Experimental Psychology: General, 109, 75-97.

Eriksen, C. W., \& Collins, J. F. (1967). Some temporal characteristics of visual pattern perception. Journal of Experimental Psychology, $74,476-484$.

Eriksen, C. W., \& Collins, J. F. (1968). Sensory traces versus the psychological moment in the temporal organization of form. Journal of Experimental Psychology, 77, 376-382.

Feldman, J. A. (1985). Four frames suffice: A provisional model of vision and space. Behavioral and Brain Sciences, 8, 265-289.

Hochberg, J. (1968). In the mind's eye. In R. N. Haber (Ed.), Contemporary theory and research in visual perception (pp. 309331). New York: Holt, Rinehart \& Winston.

Irwin, D. E., \& Brown, J. S. (1987). Tests of a model of informational persistence. Canadian Journal of Psychology, 41, 317-338.

Irwin, D. E., Yantis, S., \& Jonides, J. (1983). Evidence against visual integration across saccadic eye movements. Perception \& Psychophysics, 34, 49-57.
Irwin, D. E., \& Yeomans, J. M. (1986). Sensory registration and informational persistence. Journal of Experimental Psychology: Human Perception and Performance, 12, 343-360.

Jonides, J., Irwin, D. E., \& Yantis, S. (1982). Integrating visual information from successive fixations. Science, 215, 192-194.

Jonides, J., Irwin, D. E., \& Yantis, S. (1983). Failure to integrate information from successive fixations. Science, 222, 188.

Kahneman, D., \& Treisman, A. (1984). Changing views of attention and automaticity. In R. Parasuraman \& D. R. Davies (Eds.), Varieties of attention (pp. 29-61). New York: Academic Press.

Matin, L. (1972). Eye movements and perceived visual direction. In D. Jameson \& L. M. Hurvich (Eds.), Handbook of sensory physi$\operatorname{ology}$ (Vol. 7, No. 4, pp. 331-379). Berlin, FRG: Springer-Verlag.

McConkie, G. W., \& Rayner, K. (1976). Identifying the span of the effective stimulus in reading: Literature review and theories of reading. In H. Singer \& R. B. Ruddell (Eds.), Theoretical models and processes of reading (pp. 137-162). Newark, DE: International Reading Association.

McConkie, G. W., \& Zola, D. (1979). Is visual information integrated across successive fixations in reading? Perception \& Psychophysics, 25, 221-224.

McConkie, G. W., Zola, D., Blanchard, H. E., \& Wolverton, G. S. (1982). Perceiving words during reading: Lack of facilitation from prior peripheral exposure. Perception \& Psychophysics, 32, 271281.

McRae, K., Butler, B. E., \& Popiel, S. J. (1987). Spatiotopic and retinotopic components of iconic memory. Psychological Research, 49, 221-227.

O'Regan, J. K., \& Levy-Schoen, A. (1983). Integrating visual information from successive fixations: Does trans-saccadic fusion exist? Vision Research, 23, 765-768.

Pollatsek, A., Rayner, K., \& Collins, W. E. (1984). Integrating pictorial information across eye movements. Journal of Experimental Psychology: General, 113, 426-442.

Rayner, K., McConkie, G. W., \& Zola, D. (1980). Integrating information across eye movements. Cognitive Psychology, 12, 206-226.

Rayner, K., \& Pollatsek, A. (1983). Is visual information integrated across saccades? Perception \& Psychophysics, 34, 39-48.

Ritter, M. (1976). Evidence for visual persistence during saccadic eye movements. Psychological Research, 39, 67-85.

Sun, J.-S., \& Irwin, D. E. (1987). Retinal masking during pursuit eye movements: Implications for spatiotopic visual persistence. Journal of Experimental Psychology: Human Perception and Performance, $13,140-145$.

Trehub, A. (1977). Neuronal models for cognitive processes: Networks for learning, perception, and imagination. Journal of Theoretical Biology, 65, 141-169.

Ullman, S. (1984). Visual routines. Cognition, 18, 97-159.

Van der Heijden, A. H. C., Bridgeman, B., \& Mewhort, D. J. K. (1986). Is stimulus persistence affected by eye movements? A critique of Davidson, Fox, and Dick (1973). Psychological Research, 48, 179-181.

White, C. W. (1976). Visual masking during pursuit eye movements. Journal of Experimental Psychology: Human Perception and Performance, 2, 469-478.

Wolf, W., Hauske, G., \& Lupp, U. (1978). How pre-saccadic gratings modify post-saccadic modulation transfer functions. Vision Research, 18, 1173-1179.

Wolf, W., Hauske, G., \& Lupp, U. (1980). Interactions of pre- and post-saccadic patterns having the same coordinates in space. Vision Research, 20, 117-125.

Received November 16, 1987

Revision received February 22, 1988

Accepted February 24, 1988 